Musées, Patrimoine et Culture scientifiques et techniques

157 | 2015

janvier-février 2015

\title{
Quand le musée soigne
}

\section{Axelle Marin}

\section{OpenEdition \\ Journals}

Édition électronique

URL : http://journals.openedition.org/ocim/1471

DOI : 10.4000/ocim.1471

ISSN : 2108-646X

Éditeur

OCIM

Édition imprimée

Date de publication : 1 janvier 2015

Pagination : 12-17

ISSN : 0994-1908

Référence électronique

Axelle Marin, "Quand le musée soigne », La Lettre de l'OCIM [En ligne], 157 | 2015, mis en ligne le 01 janvier 2016, consulté le 10 décembre 2020. URL : http://journals.openedition.org/ocim/1471 ; DOI : https://doi.org/10.4000/ocim.1471

Ce document a été généré automatiquement le 10 décembre 2020.

Tous droits réservés 


\title{
Quand le musée soigne
}

\author{
Axelle Marin
}

L'exposition Premier pas 2014 présentait des œuvres de patients de l'hôpital Victor Jousselin, réalisées au musée d'Art et d'Histoire de Dreux, à la chapelle de l'Hôtel-Dieu, en plein centre-ville de Dreux.

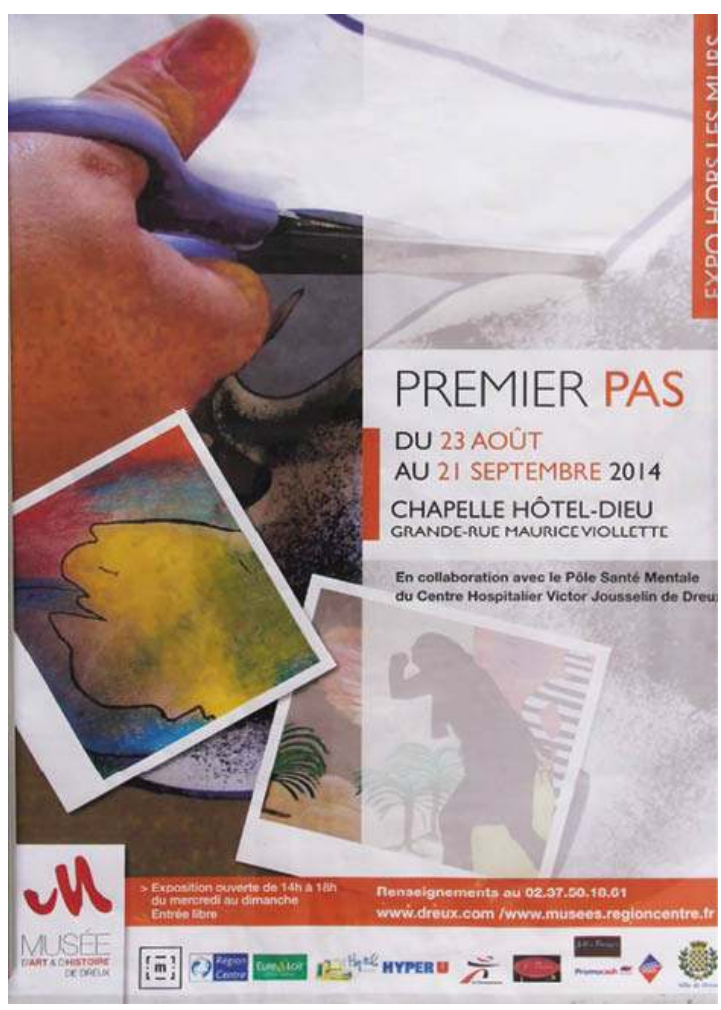

(c) Musée d'Art et d'Histoire, Ville de Dreux/AR

1 Lorsque l'on parle d'art-thérapie, des exemples comme celui d'Antonin Artaud s'imposent d'emblée à l'esprit avec tout le foisonnement, la violence et le génie du personnage. Il est en effet l'un des exemples les plus connus en France de patients soignés avec l'aide de l'art-thérapie. En 1936, il est atteint de troubles qui le conduisent à l'enfermement en 
hôpital psychiatrique dès l'année suivante. Il souffre d'hallucinations et d'idées de persécution. Il est transféré en 1938 à l'Hôpital Sainte-Anne à Paris où le certificat de quinzaine du 15 avril indique: "Prétentions littéraires peut-être justifiées dans la limite où le délire peut servir d'inspiration. À maintenir". Après plusieurs transferts jusqu'à Rodez, ayant subi des traitements à l'électrochoc, il est hébergé dans une clinique d'Ivry-sur-Seine où il est libre des ses mouvements. Durant cette période, il écrit, dessine, peint et crée plus de deux cents œuvres. Certes, il dessinait dès 1918, mais ces moments de thérapie sont en soi une inspiration et la création (notamment dans les dessins demandés et analysés par ses psychiatres) est un support à la guérison.

2 Cet exemple si particulier de traitement par l'art est une forme de caution intellectuelle et culturelle car, encore aujourd'hui, il est difficile de comprendre comment l'art, qui élève et éduque l'esprit, pourrait aussi soigner. Quant à la notion de création par des patients qui n'ont aucune formation artistique (outre le fait qu'ils sont dans une situation psychique affaiblie sinon hors norme), elle est souvent niée par le monde des arts. C'est oublier l'art brut ; c'est a contrario prendre en compte l'exceptionnalité du talent.

Bien loin de croire que les patients de l'Hôpital psychiatrique de Dreux aient tous des dispositions artistiques poussées et soient des créateurs qui s'ignorent, loin de les considérer comme des enfants qui copient pour appréhender et peu à peu maîtriser, l'équipe du musée d'Art et d'Histoire de Dreux, en partenariat avec le service Psychiatrie de l'Hôpital Victor Jousselin, a mis en place des ateliers permettant à des patients grands dépressifs, voire atteints de troubles psychotiques, à la fois de verbaliser ou exprimer leur mal-être et leur espoir, mais également de reprendre pied dans une vie sociale normale, par la fréquentation d'un lieu ouvert qui ne soit plus l'Hôpital et par une certaine forme de contractualisation, tant leur absence aux séances est une remise en cause de tout le groupe.

\section{Les prémices}

L'expérience Premier pas qui existe au musée d'Art et d'Histoire de Dreux depuis trois ans se rapproche donc de l'art-thérapie sans en être véritablement un exemple parfait car les ateliers sont encore ponctuels et ne voient pas l'évolution artistique des patients sur plusieurs années. Mais on ne peut l'expliquer sans évoquer les fondements de ce courant, qui commença tardivement en Europe.

5 Ainsi, à l'asile de Bicêtre à Paris, jusqu'à la fin du XVIII siècle, les "fous" étaient enfermés avec les criminels. La première étape de la prise en compte de la maladie en tant que telle et non comme une déviance dangereuse pour la société fut la décision du médecin chef Philippe Pinel en 1794 de ne plus les enchaîner. En parallèle, le docteur Pinel préconisa des ateliers occupationnels dans les nouveaux espaces de soins, avec pour but de distraire les malades et de les éloigner de leur délire et de leur agitation. Ces ateliers virent ainsi la production d'objets, "œuvres", qui furent parfois conservés par les médecins aliénistes et constituèrent le début des collections, surtout à la fin du XIX siècle. En 1872, Ambroise Tardieu, président de l'Académie nationale de Médecine, doyen de la faculté de Médecine et professeur de Médecine légale à l'université de Paris, publia la première reproduction d'un dessin de fou, dans le traité de médecine légale : Étude médico-légale sur la folie. 
Préparation de l'exposition Premier pas 2014 par les patients au sein des ateliers pédagogiques du musée d'Art et d'Histoire de Dreux

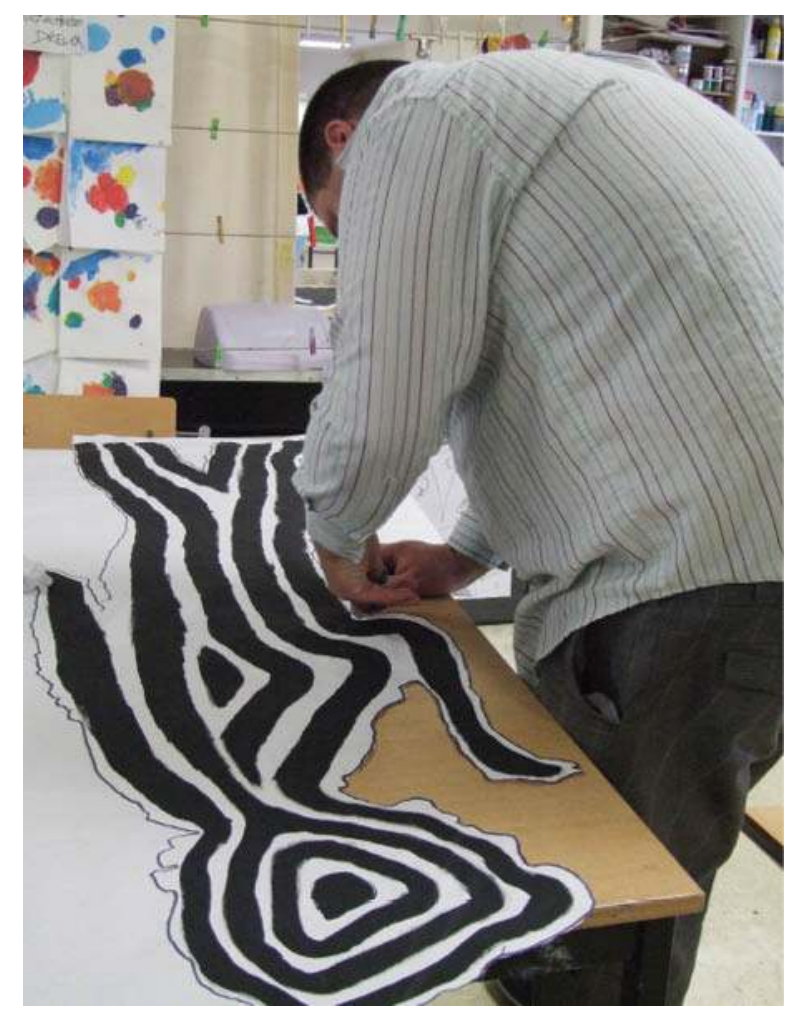

(C) Musée d'Art et d'Histoire,

Ville de Dreux/AR

En 1900 à Londres, la première exposition d'art psychopathologique eut lieu au Bethlem Royal Hospital. Il s'agissait de la première exposition de peinture constituée d'œuvres de malades mentaux. En 1905, à Paris, le docteur Auguste Marie réalisa la première exposition française et créa le "petite musée de la folie" à Villejuif. L'exposition de Marie servit de support à la publication du docteur Meunier, alias Marcel Réja, L'Art malade, constituée de dessins de fous et premier ouvrage qui reconnaissait clairement une valeur esthétique aux productions des "aliénés". C'était là un "grand pas vers la reconnaissance de l'homme derrière le malade"1. Walter Morgenthaler, psychiatre allemand, publia en $1921 \mathrm{~A}$ Psychiatric Patient as Artist, un livre de référence sur l'art psychopathologique. En 1922, il publia une monographie d'Adolf Wölfli, patient qui avait réalisé une très grande quantité de dessins et collages. La même année, Hans Prinzhorn publia l'ouvrage Expression de la folie. Dessin, peinture, sculpture d'asile dans lequel il étudiait des productions (cinq mille dessins) de patients hospitalisés en psychiatrie. L'ouvrage connut un réel succès dans toute l'Europe.

7 La notion d'art brut, due au peintre français Jean Dubuffet, est, quant à elle, apparue en 1945. Il faut entendre par là "des œuvres exécutées par des personnes sans culture, en marge de la société, ou internées dans des hôpitaux psychiatriques. Ces œuvres [sont] étrangères au champ artistique institué et radicalement autodidactes"2. Invité avec d'autres artistes français par l'office national suisse du tourisme à un voyage en Suisse au lendemain de la Seconde Guerre mondiale, Dubuffet découvrit les œuvres de Louis Soutter et d'Aloïse (en Suisse romande), d'Adolf Wölfli et d'Heinrich Anton Müller (collection du psychiatre Walter 
Morgenthaler constituée de plusieurs milliers de créations d'artistes schizophrènes internés à l'asile de Waldau dans le canton de Berne). Ce voyage fut essentiel car Dubuffet se mit dès lors, et afin de les collectionner, à chercher dans toute l'Europe ces œuvres en marge de la culture institutionnelle. En 1947, il créa le Foyer de l'art brut à Paris, puis la Compagnie de l'art brut, dont firent partie André Breton et Jean Paulhan. Il organisa sa première exposition sur l'art brut, L'Art brut préféré aux arts culturels, en 1949 à la galerie Drouin à Paris.

Les patients et l'équipe soignante produisent tous des travaux, qui sont soit anonymes, soit signés d'un pseudonyme qu'ils choisissent, ce qui est le cas de "Patrice".

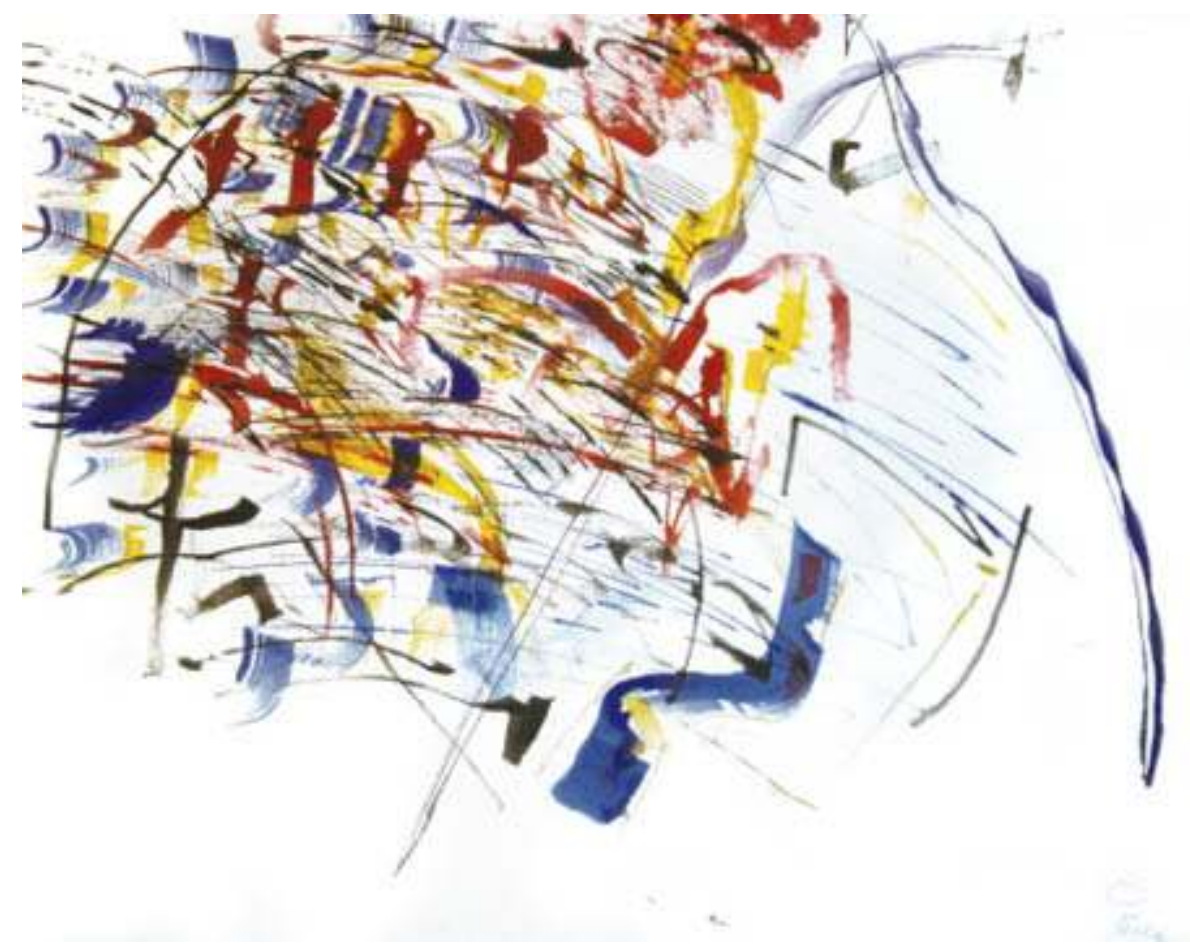

(c) Musée d'Art et d'Histoire, Ville de Dreux/AR peintre britannique Adrian Hill contracta la tuberculose en 1938, il fut soigné en sanatorium, où, alité, il dessina les objets à portée de la main, sa chambre, son opération. Il donna en 1945 le nom d'Art Therapy à ce qu'il avait mis en place et qui fut quasiment immédiatement encouragé par les plus hautes instances: hôpitaux, Croix-Rouge, ministère de la Santé britannique... C'était là la naissance d'un terme qui a fait date : l'artthérapie. Une première exposition eut lieu à l'hôpital Sainte-Anne de Paris en 1946 puis une deuxième en 1950, exposition internationale d'art psychopathologique en lien avec le premier congrès mondial de psychologie. Ces deux événements furent l'occasion de la reconnaissance et de l'introduction de la pratique artistique avec des objectifs thérapeutiques dans les hôpitaux français. Les collections d'art brut se sont dès lors développées et l'art-thérapie s'est institutionnalisé, jusqu'à être aujourd'hui une discipline reconnue et enseignée à l'université.

Ces prémices sur l'art et l'aliénation sont essentiels dans le cadre des ateliers du musée de Dreux, mais les missions des musées et leur ouverture à tous les publics le sont tout autant. En effet, l'expérience Premier pas tire avant tout ses fondements de l'ouverture des 
musées à tous les publics, réaffirmée dans la loi $\mathrm{n}^{\circ} 2002-5 \mathrm{du} 4$ janvier 2002 dite "loi Musées de France" et qui implique que les Musées de France doivent "concevoir et mettre en ceuvre des actions d'éducation et de diffusion visant à assurer l'égal accès de tous à la culture". L'article L441-2 du Code du Patrimoine indique ainsi que "Les musées de France ont pour missions permanentes de:

a) Conserver, restaurer, étudier et enrichir leurs collections;

b) Rendre leurs collections accessibles au public le plus large ;

concevoir et mettre en cuvre des actions d'éducation et de diffusion visant à assurer l'égal accès de tous à la culture;

d) Contribuer aux progrès de la connaissance et de la recherche ainsi qu'à leur diffusion".

Dès lors, tous les publics potentiels doivent être pris en compte, y compris ceux qui ne peuvent pas se rendre dans un musée en raison de leur enfermement, prisonniers ou hospitalisés par exemple, publics dits "empêchés". Le musée de Dreux, au début du projet, n'était en effet pas d'emblée dans une démarche d'art-thérapie mais bien d'abord dans l'accomplissement de ses missions. L'équipe du musée travaillait alors depuis plusieurs années avec grand succès avec les écoles maternelles et primaires environnantes, mais les adultes étaient peu pris en compte et le travail sur les populations en difficulté était à faire.

Exposition Premier pas 2014 : œuvres sur la notion du corps.

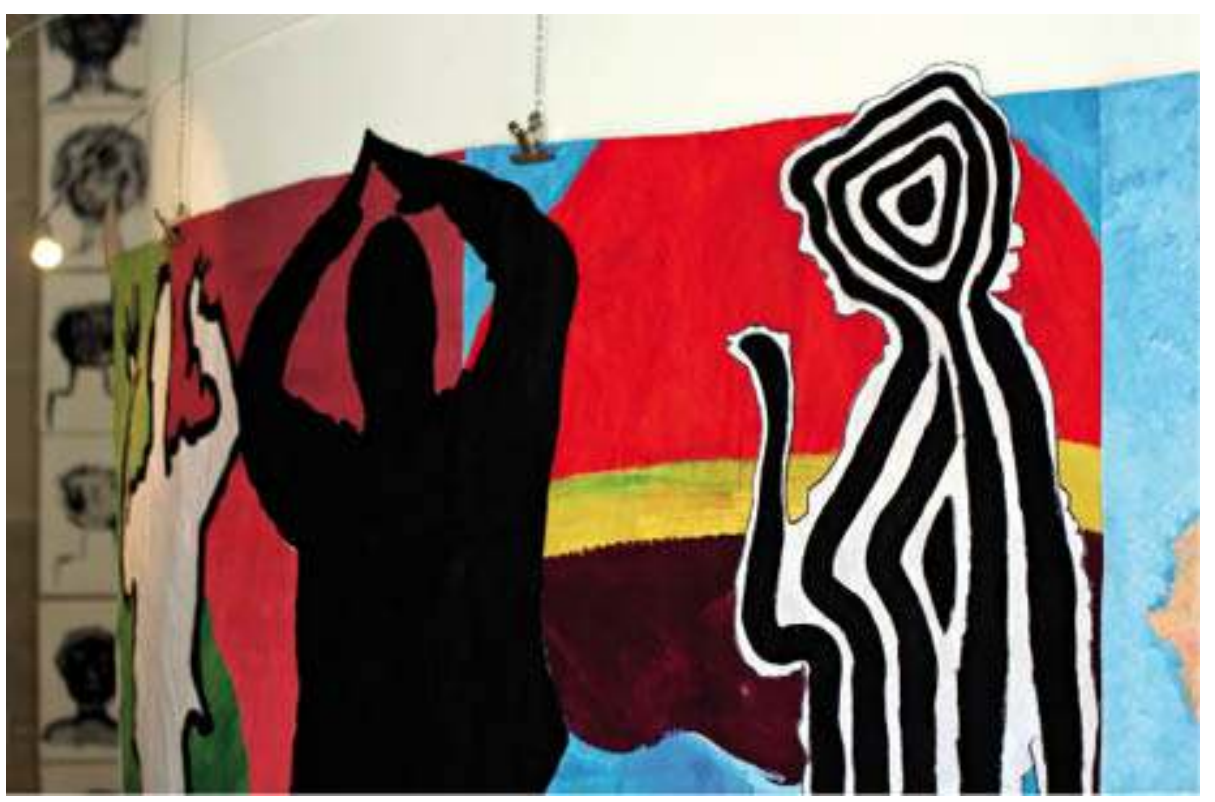

(c) Musée d'Art et d'Histoire, Ville de Dreux/AR

Ainsi que l'indique le rapport d'information sur les musées déposé à l'Assemblée Nationale par la Commission des affaires culturelles, familiales et sociales : "on ne peut plus se contenter d'attendre que les populations en difficulté franchissent seules les obstacles symboliques, et pas seulement financiers comme on le pense trop facilement, qui les coupent des musées. C'est donc la confrontation, à l'extérieur, avec les exigences d'un public différent, qui permettra à ces institutions d'évoluer et de modifier leurs politiques de publics" ${ }^{3}$.

16 Au musée de Dreux, un dispositif global, dénommé "Ensemble" permit alors de mettre en place à la fois des ateliers pour des associations d'adultes en difficulté ou des visites 
destinées à des personnes en situation de handicap. L'idée d'ouvrir les ateliers aux patients de la section Psychiatrie de l'Hôpital Victor Jousselin de Dreux est peu à peu née de cette volonté de rendre le musée accessible à tous.

Elle n'aurait cependant pas pu aboutir sans l'adhésion immédiate de l'équipe du service Psychiatrie de l'Hôpital, où des ateliers d'ergothérapie et d'art-thérapie avaient déjà lieu et dont la bienveillante écoute avait été préparée par plus de cent ans de réflexion sur "l'art des fous". "Le terme d'art-thérapie, qui fut créé lors de l'invention de ces méthodes de soin, est fort pratique et communément utilisé [...]. Cependant, il induit des confusions quant aux définitions qui peuvent en être données. En effet, ce n'est pas l'art en lui-même qui est susceptible de soigner, c'est-à-dire d'induire un processus de changement, mais c'est une pratique artistique mise en cuvre selon des modalités bien définies. En cela, la dénomination de psychothérapie à médiation artistique est beaucoup plus juste et adaptée" ${ }^{4}$. C'est en effet plus modestement cette médiation artistique et surtout cette collaboration entre deux structures publiques qu'il faut, dans le dispositif de Dreux, mettre en avant.

Cette collaboration posait cependant quelques soucis. Comment mettre en sécurité dans un lieu public des patients ordinairement enfermés, ne sortant que sur prescription médicale, voire avec un accord préalable du Préfet et les réintégrer dans un ensemble de visiteurs classiques? Quelle médiation créer pour eux au sein d'un musée qui n'avait pas encore ce type d'expériences? Quel risque pour les autres visiteurs et pour les collections?

\section{Le déroulement}

19 Ainsi, des patients de la section Psychiatrie générale de l'Hôpital Victor Jousselin de Dreux viennent régulièrement au musée et participent à des ateliers de création et d'échanges. Depuis septembre 2013, un groupe de l'Hôpital de Jour - composé de patients schizophrènes rentrés chez eux mais qui reviennent pour des soins réguliers - fait de même.

20 Ces patients sortent donc du cadre de l'Hôpital, ce qui fait la spécificité drouaise car, d'ordinaire, ce sont les thérapeutes et les médiateurs qui viennent pour animer des ateliers dans l'enceinte de l'Hôpital.

21 Les patients sont encadrés par le personnel soignant qui les accompagne au quotidien dans leur lutte contre la dépression et les maladies névrotiques et psychotiques (infirmiers psychiatriques, ergothérapeutes, psychomotriciens), le tout sous le contrôle, au retour à l'Hôpital, des médecins psychiatres du service.

22 Au musée, ils sont pris en charge en toute discrétion et à un moment où l'établissement est normalement fermé au public, confidentialité oblige, par une médiatrice culturelle, qui les aide à s'exprimer à la fois sur les collections du musée et sur des créations toutes personnelles. Elle est dès lors un "métacréateur" : "accompagnateur d'autrui sur les chemins de la création comme de la réalisation de soi" ${ }^{15}$. Médiatrice culturelle depuis une dizaine d'années au début du projet, l'intervenante désignée connaissait surtout le travail avec des scolaires et quelques groupes d'adultes. Ses qualités humaines et professionnelles indéniables étaient nécessaires à la réussite rapide de ce projet. Il lui a d'abord été proposé, fortement suggéré et son acceptation se fit à la fois avec respect, conscience du défi à relever et une pointe d'angoisse de ne pas savoir comment aborder ces visiteurs hors norme. Cependant, des expériences précédentes avaient déjà permis de la 
familiariser (ainsi que l'équipe complète du musée) avec des publics spécifiques. Elle accueillait en effet déjà des personnes mal ou non voyantes ainsi que des jeunes issus d'instituts médico-éducatifs ou de classes adaptées. Son sens de l'écoute et sa bienveillance liés au respect du cadre et de la règle étaient déjà un point essentiel qui permettrait aux patients de ne pas être déstabilisés mais de voir leur créativité contenue, accompagnée.

Euvre réalisée en relation avec l'exposition Portraits peints portraits gravés du musée d'Art et d'Histoire.

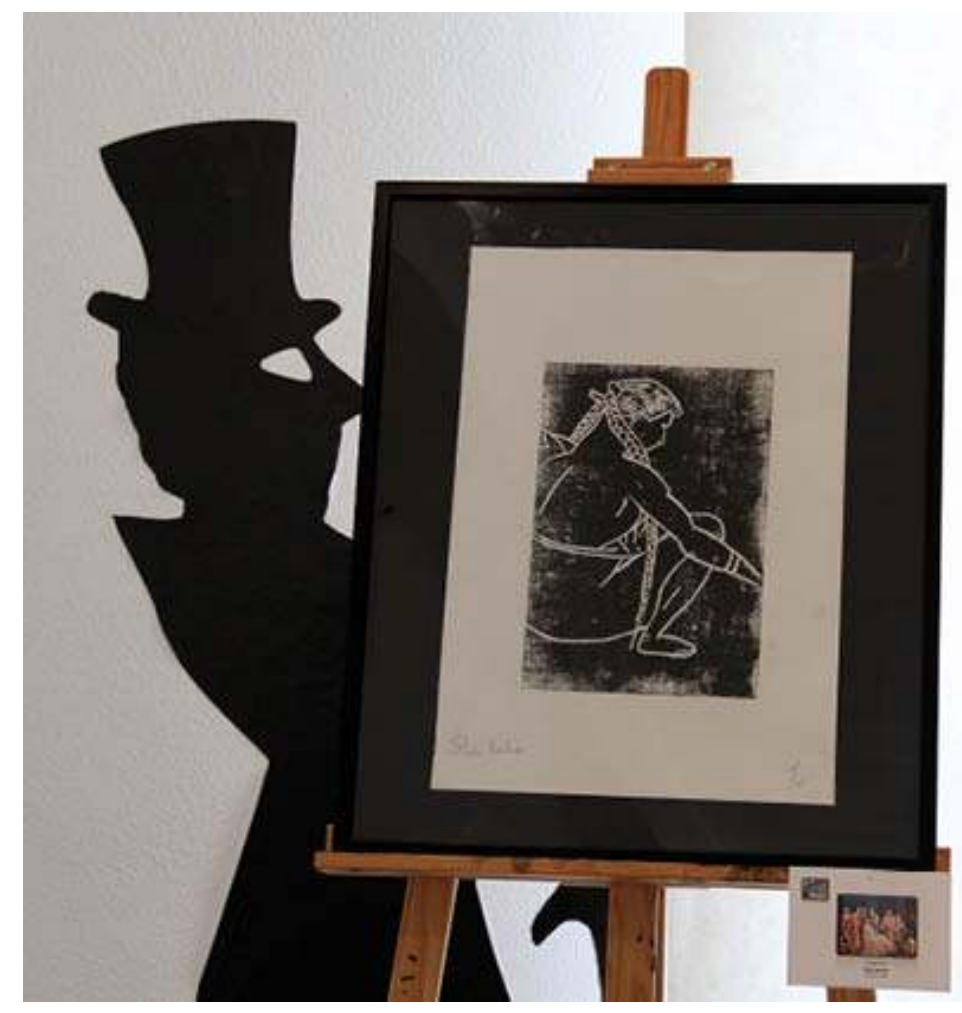

(c) Musée d'Art et d'Histoire,

Ville de Dreux/AR

Autour d'une œuvre, dans les salles, le discours vient de la médiatrice puis petit à petit un échange, une conversation se créent avec et entre les patients. C'est le début, l'apprivoisement du lieu et de la médiatrice par le groupe. Vient ensuite le temps de l'exercice en atelier, qui va lui aussi participer à la mise en confiance et faciliter la lecture d'une œuvre ainsi que l'approche d'une technique. Enfin, arrive le temps de l'interprétation et de la démarche créative. Elle se fait toujours sur des œuvres du musée, peintes ou sculptées, mais la musique vient parfois, les yeux fermés, accompagner l'acte créatif.

Comme l'indique le docteur Anne-Marie Dubois, psychiatre et responsable de l'unité artthérapie du Centre hospitalier Sainte-Anne de Paris, "l'atelier est un espace d'expériences possibles et sûr, qui joue aussi un rôle social pour chaque participant. De son côté, [le médiateur] a conscience des évocations qui émanent de son statut et de son identité. Il connait ce domaine et reconnait l'œuvre qui est en train de se faire sous ses yeux, tout en conseillant une neutralité bienveillante; il n'est pas là pour juger mais pour tenter de comprendre la nature de ce qui se déroule, dans l'atelier dont il a la charge ${ }^{\text {"6}}$. Le médiateur au musée n'est pas un thérapeute et 
ne se prétend pas l'être. Il est un medium entre les collections du musée et les visiteurs. Le but des séances est clairement posé dès la première : permettre la création d'une exposition à la fin de la saison culturelle. Il faut donc que les patients se projettent dans le temps, alors même que, en raison de leurs pathologies, un avenir leur est souvent inconcevable. Et leur venue à chaque séance est dès lors présentée comme nécessaire pour l'évolution de tout le groupe et la réussite du projet de tous. Chaque individu retrouve ainsi une place dans une forme de société, une utilité, une raison d'être.

La date choisie pour les ateliers coïncidait avec celle de la fermeture au public, le personnel de surveillance suit en permanence le groupe. Le personnel soignant de l'Hôpital, qui encadre les patients, a l'habitude de l'accompagnement dans des actions de thérapie hors les murs telle l'équithérapie et, dans l'activité au musée, deux soignants sont présents pour quatre, voire six patients. Ce groupe est traité exactement comme tous les groupes de visiteurs. Les règles du musée (ne pas toucher, ne pas courir, ne pas déranger les autres visiteurs...) sont énoncées à chaque début de séance et doivent être respectées. L'agent de surveillance est là pour les rappeler et les faire appliquer. Elles n'ont toutefois encore jamais été enfreintes et aucun comportement qui aurait pu engendrer un risque pour autrui ou pour les collections n'a été observé jusqu'ici.

\section{Les résultats}

L'année 2011-2012 a été un test. Sur les séances, réparties tout au long de l'année, trois groupes ont pu ainsi se succéder et réaliser des "œuvres" qui ont trouvé leur place dans l'exposition finale, Premier pas, alors en juillet. Depuis, pour le service de Psychiatrie générale, un groupe de cinq patients vient toutes les semaines sur une séance de deux heures en moyenne et dans une session d'un mois environ. Le mois suivant, c'est un nouveau groupe qui prend sa place. Les patients de l'Hôpital de Jour viennent une fois tous les quinze jours avec le même groupe toute l'année. Ce groupe s'apparente donc plus aux dispositifs médicaux de l'art-thérapie, où la récurrence permet l'évolution à la fois psychique et artistique : "La régularité du cadre temporel des ateliers [...] est nécessaire afin de stimuler et d'accueillir les productions. C'est un élément fondamental en lequel le patient doit avoir une totale confiance pour exister pleinement en ce lieu"7.

Ce dispositif exceptionnel, qui fait (re)venir les patients dans un lieu que la maladie leur faisait déserter, notamment parce qu'il est public et intimidant, leur a permis de se projeter dans l'avenir, de contractualiser leur présence. Cette phase de test a été un réel succès en termes de soins et l'équipe soignante s'accorde à dire que ce projet a eu un impact réellement bénéfique sur le traitement et l'évolution de la maladie. Au musée même, la découverte de leurs propres capacités, voire de leur talent a été un moteur pour les participants. 
Euvre réalisée en relation avec l'exposition Portraits peints portraits gravés du musée d'Art et d'Histoire.

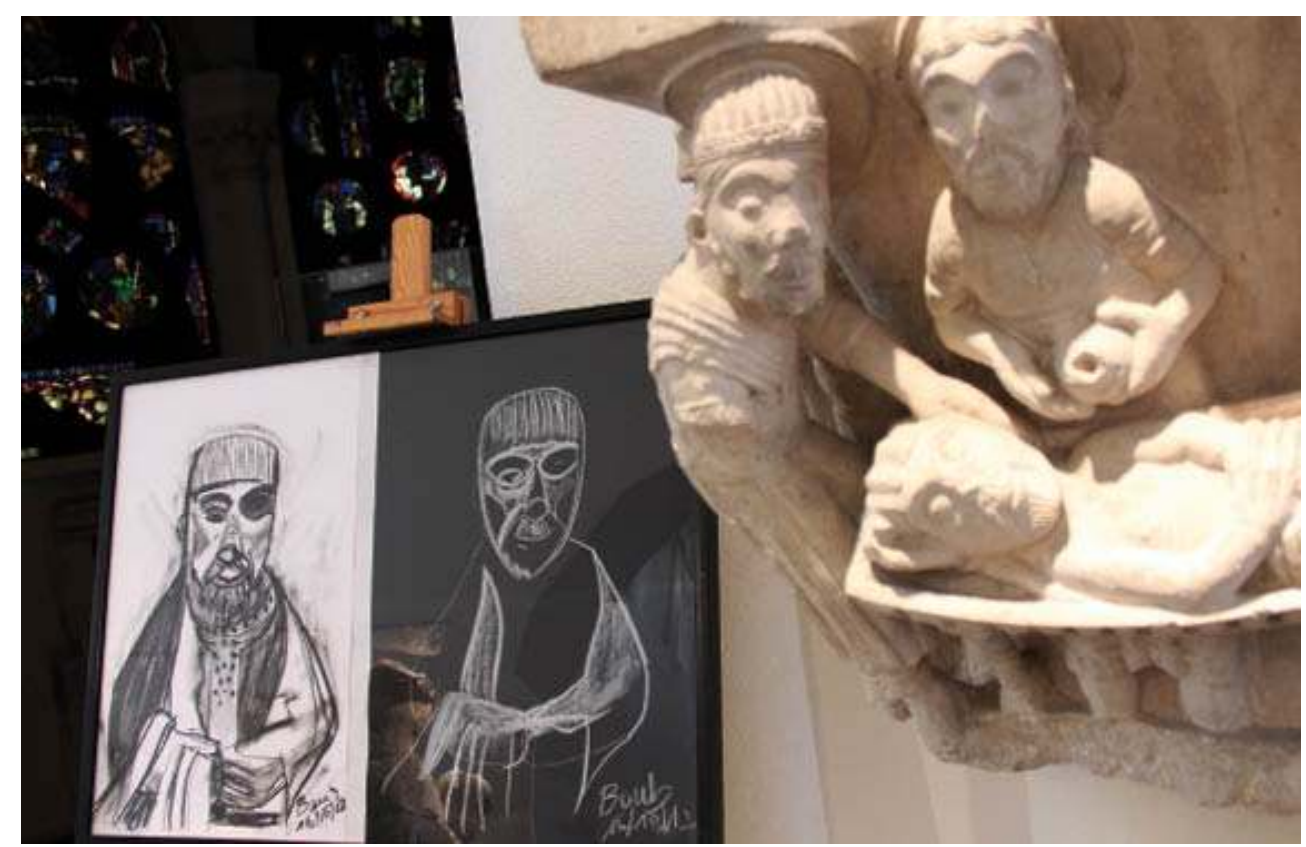

(C) Musée d'Art et d'Histoire,

Ville de Dreux/AR

Les difficultés préalables pour les patients, à savoir entrer dans un lieu public avec le risque d'être reconnu comme malade, sortir de l'espace protecteur de l'Hôpital pour entrer dans un cadre inconnu et intimidant, mais aussi les appréhensions de l'équipe du musée face à la maladie, à des réactions qui pourraient être violentes, se sont peu à peu apaisées pour laisser la place à des résultats réels.

Les patients ne se sont certes pas révélés comme de vrais artistes, aucune carrière n'est née, mais ils reconnaissent eux-mêmes le bénéfice du dispositif : réappropriation de l'espace public, prospective et vision à moyen terme de sa propre existence et non plus au jour le jour, redécouverte du plaisir, redécouverte de ce que l'on est capable de faire et de l'estime de soi, découverte des capacités d'autrui, échanges avec d'autres êtres et non plus solitude, découverte de points positifs communs, partage non uniquement de la souffrance psychologique et de la douleur mentale, voire physique mais aussi des expériences, sont autant de points positifs qui concourent au mieux-être. Il est cependant impossible de savoir si, les patients qui sont sortis de l'hôpital ont gardé une pratique artistique et ont continué à fréquenter les musées.

30 Ce dispositif fait désormais partie du Contrat Urbain de Cohésion Sociale de la Ville de Dreux. Pérenne, il prend la même forme chaque année jusqu'à l'exposition finale. Son succès amène aujourd'hui à se poser la question de son développement par d'autres actions, à commencer par la visite d'une institution muséale différente, avec l'accompagnement du personnel soignant et de l'équipe du musée, comme voyage thérapeutique, peut-être même à l'étranger.

31 Forts de cette expérience, l'Hôpital et la Ville ont depuis étendu le partenariat à un projet sur le spectacle vivant et les expositions Premier pas ont désormais lieu dans une salle en plein centre ville de Dreux, la chapelle de l'Hôtel-Dieu, poursuivant ainsi le 
décloisonnement et permettant une meilleure communication auprès du grand public autour des maladies mentales.

\section{Témoignage de Delphine Eristov intervenante en arts plastiques au musée d'Art et d'Histoire de Dreux}

\footnotetext{
"Il y a quatre ans, lorsque Axelle Marin, ma directrice m'a annoncé que nous pourrions recevoir des patients du Pôle Santé Mentale de l'Hôpital de Dreux, j'ai certainement eu une montée d'adrénaline et une énorme bouffée d'angoisse. En effet, comment allais-je pouvoir recevoir ce public si particulier, moi qui n'avais l'habitude que d'une médiation envers le jeune public? Bien sûr, je travaillais déjà depuis quelques années avec les IME, mais il s'agissait d'enfants.

Alors je me suis lancée dans l'aventure. Je me suis adaptée. J'ai d'abord utilisé mon expérience auprès $\mathrm{du}$ jeune public pour amener les patients vers les œuvres de nos collections, de façon ludique. Des jeux de détails à retrouver, des mallettes à expériences sur les sens, les couleurs et puis la musique...

Ensemble, nous apprenons à observer, nous échangeons nos sentiments, nos ressentis face aux cuvres.

Vient ensuite le moment de la création. Mes consignes sont simples pour permettre une interprétation des œuvres, avec des médiums variés.

Ce n'est pas toujours aisé, parfois certains patients sont intériorisés, d'autres sont euphoriques. Il faut composer avec chacun et que tous participent et surtout que tous trouvent un moment de répit et de réconfort lorsqu'ils sont dans notre musée, avec moi.

C'est une expérience très riche d'enseignement sur les rapports humains et sur le regard que chacun porte aux cuvres.

Aujourd'hui, c'est mon expérience avec le public dit "empêché" que j'utilise pour le jeune public".
}

\section{BIBLIOGRAPHIE}

Catalogue de l'exposition Adolf Wölfli univers, LaM, exposition du 9 avril au 3 juillet 2011 au LaM, Lille-métropole.

Dubois, A.-M. L'Art des fous à l'œuvre d'art. Paris : Histoire d'une collection, Édition du Centre d'Étude et de l'Expression, 2007.

Dubois, A.-M. Art-Thérapie- Principes, méthodes et outils pratiques. Paris : Elsevier Masson, 2013, $156 \mathrm{p}$.

Frechuret, M. L'Art médecine, Relation avec un centre hospitalier, Musées et services des publics, actes des Journées d'étude, 14 et 15 octobre 1999, Paris, École du Louvre, édités par le ministère de la Culture et de la Communication, Direction des musées de France, 291 p.

Hill, A. L'Art contre la maladie. Paris : Vigot, 1945.

Klein, J.-P. Que sais-je ? L'art-thérapie. Paris : Presses Universitaires de France, 1997, 128 p.

Klein, J.-P. L'Art contre la maladie : Hommage à l'inventeur de l'art-thérapie - Adrian Hill. Paris : Art \& Thérapie, 2009, 104 p. 
Millet, C. L'Art contemporain. Paris : Flammarion, Collection Dominos, 1997.

Patrimoine et public en rupture : un défi pour les musées, Musées et collections publiques de France, $n^{\circ} 220,1998$.

Prinzhorn, H. Expressions de la folie, Dessins, peinture, sculpture d'asile, Connaissance de l'inconscient. Paris : Gallimard, 1984.

Rapport d'information sur les musées déposé à l'Assemblée Nationale par la Commission des affaires culturelles, familiales et sociales. Paris, 25 mai 2000, 93 p.

\section{NOTES}

1. Dubois, A.-M. Art-Thérapie : principes, méthodes et outils pratiques. Paris : Elsevier Masson, 2013, p. 7.

2. D'après la définition de Dubuffet, rappelée par le site de La Collection de l'art brut de Lausanne (Suisse), www.artbrut.ch/fr/21070/collection-art-brut-lausanne.

3. Rapport d'information sur les musées déposé à l'Assemblée Nationale par la Commission des affaires culturelles, familiales et sociales, 25 mai 2000, pp. 20-21.

4. Dubois, A.-M., op. cit., p. 15.

5. Klein, J.-P. L'art contre la maladie : Hommage à l'inventeur de l'art-thérapie, Adrian Hill. Paris : Art \& Thérapie, 2009, $104 \mathrm{p}$.

6. Dubois, A.-M., op. cit., p. 47. Le terme de médiateur remplace ici « l'art-thérapeute » du texte originel.

7. Dubois, A.-M., op. cit., p. 48.

\section{RÉSUMÉS}

Après avoir rappelé les fondements historiques de l'art-thérapie, l'auteure relate l'expérience menée au musée d'Art et d'Histoire de Dreux avec la mise en place d'ateliers de psychothérapie à médiation artistique qui s'inscrivent dans le cadre d'une politique volontariste d'ouverture du musée à tous les types de publics et qui s'adressent à des patients de l'hôpital de la ville atteints de maladie névrotiques et psychotiques.

\section{INDEX}

Mots-clés : musée, art-thérapie, psychothérapie, médiation artistique

\section{AUTEUR}

\section{AXELLE MARIN}

Attachée de conservation, responsable du musée d'Art et d'Histoire de Dreux a.marin@ville-dreux.fr 